\title{
Regular audit can lead to changes in practice and better outcomes for pregnant women with HIV V Patton*1, R Fox ${ }^{1}$, R Nandwani ${ }^{1}$, A Seaton ${ }^{1}$, A MacConnachie ${ }^{1}$, M Hepburn ${ }^{2}$, E Ellis $^{2}$, H Mactier $^{2}$ and A Winter ${ }^{1}$
}

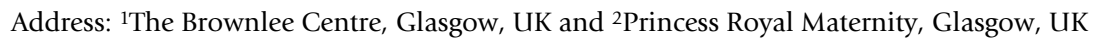

* Corresponding author

from Ninth International Congress on Drug Therapy in HIV Infection

Glasgow, UK. 9-13 November 2008

Published: 10 November 2008

Journal of the International AIDS Society 2008, I I (SuppI I):P2I 9 doi:10.II86/I758-2652-II-SI-P2I 9

This abstract is available from: http://www.jiasociety.org/content/II/SI/P219

(C) 2008 Patton et al; licensee BioMed Central Ltd.

\section{Purpose of the study}

The purpose of the study was to determine if regular audit of management of HIV-positive pregnant patients resulted in improved practice.

\section{Methods}

Over a 3-year period from 1 July 2005 to 30 June 2008, there were 50 deliveries of pregnant mothers who were HIV-positive and looked after by a joint team from the Brownlee Centre and Princess Royal Maternity, Glasgow. Each year an audit meeting reviewed all the pregnancies with a view to improving the care pathway. The audit focused on two areas which were measurable and had the potential to change practice. Changes to practice were reaudited in subsequent years.

\section{Summary of results}

Fifteen pregnancies and 10 deliveries were audited in the period 1 July 2005 to 30 June 2006, and two specific areas were identified. First, following a pre-term delivery at 24 weeks gestation, it was recognised that antiretroviral treatment for prevention of mother-to-child transmission (PMTCT) should be initiated sooner than around 26 weeks. Secondly, therapeutic drug monitoring (TDM) should be performed in patients on protease inhibitors (PIs) early in the third trimester to ensure adequate levels. In 2005-06 the mean gestation for starting antiretroviral treatment for PMTCT was 26 weeks; in 2006-07 it was 24 weeks; and in 2007-08 it was 22 weeks with one patient who presented late starting at 38 weeks. TDM was per- formed in the 3rd trimester on $0(0 \%)$ patients in $2005-$ 06 , in six (50\%) patients in 2006-07 and in 19 (95\%) patients on PIs in 2007-08. Twenty-one (84\%) had levels greater than estimated minimum trough concentration (EMTC), two (8\%) had borderline levels equal to EMTC, and two (8\%) had undetectable levels. As a result, two $(8 \%)$ with borderline levels had their doses increased (one lopinavir and one saquinavir) and both had undetectable viral loads at the time of delivery. Suspected nonadherence was confirmed in the other two patients who had undetectable PI levels.

\section{Conclusion}

Annual audit of all pregnant patients was beneficial in highlighting areas where improvements could be made to the care pathway. Antiretroviral treatment for PMTCT is now initiated at an earlier gestation to ensure undetectable viral load in case of pre-term labour. TDM has enabled doses of PIs to be increased if necessary to ensure viral load is undetectable at delivery. It has also confirmed suspected non-adherence with medication enabling other action to be taken prior to delivery to reduce risk of MTCT. 